\title{
Composite Insulator Defect Identification Method Using Improved RCNN Convolution Kernel
}

\author{
Xinhai LI ${ }^{\text {a, }}{ }^{\text {, }}$ Qifeng $\mathrm{LUO}^{\mathrm{a}}$, Qingzhu ZENG ${ }^{\mathrm{a}}$, Xinxiong ZENG ${ }^{\mathrm{a}}$, Chao YAN ${ }^{\mathrm{a}}$, Kai \\ ZHANG $^{\mathrm{b}}$ \\ ${ }^{a}$ Zhongshan Power Supply Bureau of Guangdong Power Grid Co., Ltd., Zhongshan, \\ Guangdong, China \\ b School of Electricity South China University of Technology, Guanzhou, Guandong, \\ China
}

\begin{abstract}
The detection of composite insulator defects in substations still relies on manual inspection. In this paper, we propose a detection method for insulator crack shape features by improving the RCNN convolution kernel. The method can meet the premise of insufficient training sample data, but also can get better CNN training effect, and finally achieve accurate crack recognition. In the training phase, the RGB three-channel decomposition method is used to expand the training data set; the median filtering method is used to remove the noise; the improved convolutional kernel is used to train the $\mathrm{CNN}$; in the test phase, the images are decomposed by RGB three-channel decomposition and input to CNN to get the exact crack center coordinates and length; the NMS algorithm is used to de-weight the images to get the final crack recognition results. The example analysis shows that the method in this paper can still achieve good recognition accuracy and accurately identify the specific location of cracks under the premise of insufficient training samples.
\end{abstract}

Keywords. Insulator defect identification, image processing, R-CNN; RGB threechannel filtering

\section{Introduction}

In daily operation of substations, composite insulators are prone to insulating jacket cracks due to external natural environment and long-term use aging. Such defects, if not detected and treated in time, may lead to insulator damage and bursting, resulting in protection tripping and even serious accidents such as large area blackouts in the power supply area, causing serious losses to the power system as well as the national economy [1].

Composite insulators are a very large number of outdoor substation equipment that rely on the naked eye to distinguish them, which is a large workload and also difficult to distinguish the presence of cracks. The traditional methods of insulator crack defect detection mainly include: direct observation method with regular sampling of equipment

1 Corresponding Author, Xinhai LI, Nr. 5, Xihe East Road, West District, Zhongshan City, Provincie Guangdong; E-mail: zslixinhai@163.com. 
and infrared measurement method [2]. The traditional method relies on manual, but the manual detection method is inefficient and work-intensive, and the long time observation is likely to cause visual fatigue and lead to missed detection. At the same time, infrared temperature measurement and detection method also has poor stability and reliability, low temperature measurement accuracy, poor anti-interference ability and measurement parameters are very easy to drift and other disadvantages. With the application of intelligent algorithms such as deep learning becoming mature, automatic detection of insulator crack defect characteristics using image recognition methods has become possible. The use of image recognition methods can enhance the automation and intelligence of substation operation [3], greatly reduce the reliance on manual labor for insulator crack detection, and improve the safety and stability of transmission and distribution links.

Based on the actual insulator crack image acquisition, this paper improves the CNN convolution kernel and designs a set of R-CNN for composite insulator crack feature detection method. It is demonstrated the proposed detection method with the improved R-CNN convolutional kernel can achieve an accuracy of $99.6 \%$ for the detection of cracks with a small amount of image sample data. This contributes to the realization of non-contact, online monitoring of insulator crack defects and provides new ideas for fault diagnosis of transformer operating insulators, unmanned substations, and intelligent identification of cracked insulators by robots.

\section{Overview of the Method and Improvement of the Convolution Kernel}

\subsection{Methodology Overview}

The specific process is shown in figure 1 .

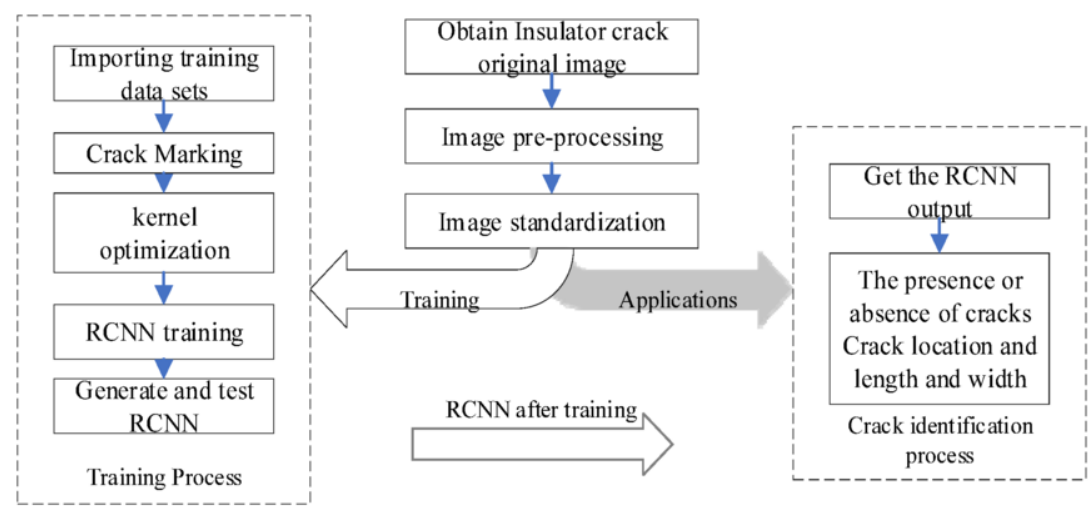

Figure 1. The R-CNN network model training and flow chart of insulator cracking image recognition

First, the acquired color images are decomposed into three single-channel insulator images of R, G, and B according to the image preprocessing method described in Section I; subsequently, median filtering is used to remove the noise adulterated in the acquisition and transmission process of this image; then, the processed 3-channel images are 
normalized to generate input data that meet the requirements of R-CNN and are input into the network for prediction; finally, the sets of pixels of $\mathrm{R}, \mathrm{G}$, and If the average confidence level exceeds the threshold set by the algorithm, the Mask network can be used to obtain information about the location, length and width of the crack.

\subsection{Convolution Kernel Improvement for Insulator Cracks}

The complex CNN brings more computational resources consumption, and when the parameters in the kernel matrix cannot be fully utilized due to the unreasonable design of the convolutional kernel, it will lead to a large amount of wasted computational resources. In the substation, for the insulator image file collected by the inspection "robot", its pixels are determined, and the relative size of the insulator in the image is also relatively fixed, and the relative size of the crack (pixel point and length and width) is also basically determined. According to this feature of the crack image, the convolution kernel can be designed by specifying the number of rows and columns of the convolution kernel, as shown in figure 2, to reduce the demand for computational resources for crack recognition and improve the training speed and recognition accuracy of $\mathrm{CNN}$. The specific procedure is as follows.

1. Pick $n$ typical pictures of cracks and frame the cracks in the figure

2. Obtain the aspect pixel points of the selected cracks $r_{k} \times c_{k}$ and construct the aspect set.

$$
R=\left\{r_{1}, r_{2}, \ldots, r_{n}\right\}, C=\left\{c_{1}, c_{2}, \ldots, c_{n}\right\}
$$

3. Considering that the $r$ of the crack to be identified is relatively fixed, in order to reduce the number of convolution kernel parameters, it is taken as

$$
r_{\text {kernel }}=\min (R), c_{\text {kernel }}=\min (C)
$$

As the length and width pixels of the designed convolution kernel. As the crack in figure 2 , the shortest crack can be covered by selecting a convolution kernel of $7 \times 17$. 


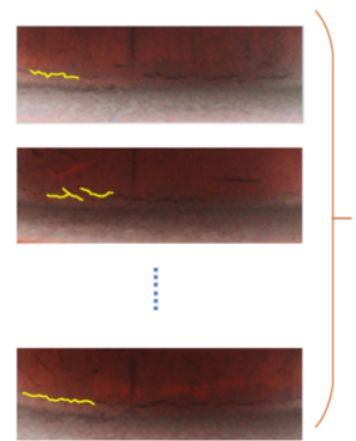

a). 挑选常见绝缘子裂统

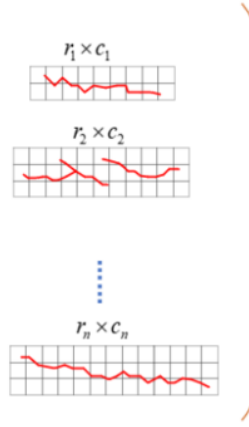

b). 框选烈纹长宽(像素)

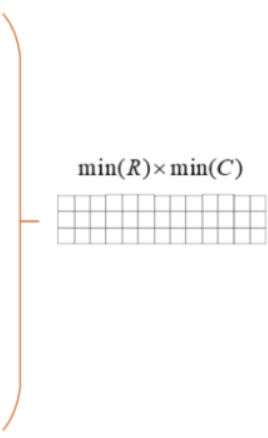

c).卷积核长宽(像素)

a-Common insulator cracks b-Selected crack length c-Convolution kernel

Figure 2. Convolution kernel design for insulator cracks

\section{Insulator Image Pre-processing}

Since neural networks are prone to the problems of poor network generalization and network overfitting when the number of samples in the training set is small. Therefore, to solve the problem of small number of insulator samples, the single insulator color image is decomposed into R, G and B channel images before image pre-processing, and the images are processed by image processing algorithms such as rotation and panning to expand the sample set. At the same time, external factors such as the camera lens production process and the lighting environment can cause the insulator image data to contain a lot of noise, and this noise can easily affect the accuracy of the R-CNN network model in training and prediction, and may cause a false diagnosis in serious cases. Therefore, the captured raw insulator image data needs to be filtered to eliminate the noise and enhance the contrast of the image. Commonly used filtering methods include Gaussian filtering, median filtering, recursive filtering, etc. [4] In summary, the specific process of preprocessing for the original insulator images is described as follows: first, the captured color insulator images are decomposed into three single-channel images, R, $\mathrm{G}$, and B. Subsequently, median filtering is applied to remove the noise adulterated in the acquisition and transmission process of this image, and the filtered images are normalized to obtain the unlabeled image training set as shown in figure 3 .

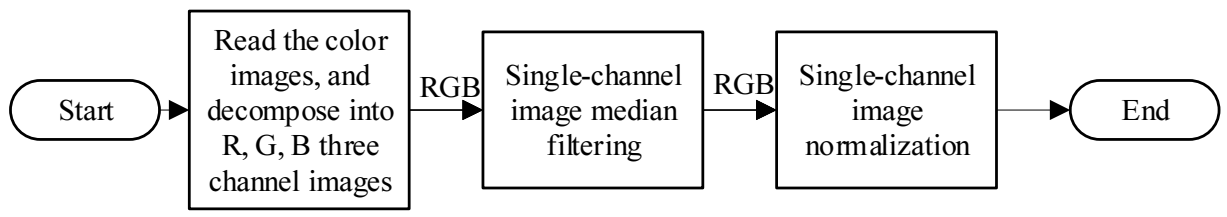

Figure 3. Flow chart of insulator image preprocessing 


\section{RCNN Design and Training}

The collected insulator crack pictures are all $512 \times 512 \times 3$ (color pictures with 512 pixels in length, 512 pixels in width, and 3 color channels). RCNN needs to accomplish two judgment tasks: 1) on a certain picture, whether there is a crack; 2) if there is a crack on a certain picture, what is the location and the length and width of the crack. Therefore, the training of the network can be regarded as a bi-objective optimization problem with an objective function of :

$$
\min : L\left(\left\{p_{i}\right\},\left\{t_{i}\right\}\right)=\frac{1}{N_{c l s}} \sum_{i=1}^{N_{c l s}} L_{c l s}\left(p_{i}, p_{i}^{*}\right)+\frac{1}{N_{\text {reg }}} \sum_{i=1}^{N_{\text {reg }}} L_{\text {reg }}\left(t_{i}, t_{i}^{*}\right)
$$

Among them :

$$
L_{c l s}\left(p_{i}, p_{i}^{*}\right)=p_{i} \log \left(p_{i}^{*}\right), L_{r e g}\left(t_{i}, t_{i}^{*}\right)= \begin{cases}0.5\left(t_{i}-t_{i}^{*}\right) & \text { if }\left|t_{i}-t_{i}^{*}\right|<1 \\ \left|t_{i}-t_{i}^{*}\right|-0.5 & \text { otherwise }\end{cases}
$$

In the above equation: $\left\{p_{i}\right\},\left\{p_{i}^{*}\right\}$ Network output classification, desired network output classification. $\left\{t_{i}\right\},\left\{t_{i}^{*}\right\}-$ Crack location of network output, desired crack location of network output. $N_{c l s} \longrightarrow$ Number of categories. $N_{\text {reg }}$ - The number of pixel points output. $L_{c l s}(\bullet) \longrightarrow$ A cross-entropy function for classification. $L_{r e g}(\cdot)-$ The smoothing function used for regression.

The parameters of the designed CNN network are shown in table 1.4 convolutional layers and 7 fully connected layers are designed, where the FC3-Classifier uses the result generated by SoftMax as the score of the input image on the crack, and the FC3Regressor uses the center and the length and width of the bounding box as the score of the crack localization. Among them, the convolution kernel of the first layer of convolution is the size of the smallest insulator crack $7 \times 17 \times 3$, and 24 convolution kernels are configured. The sliding window interval stride of each convolution kernel is set to 4 , and ReLUs is used as the neuron activation function. The parameters of each layer of convolution are obtained by convolution operation.

The training process of the whole network is divided into the following steps: I. Preprocessing the original insulator cracking images and manually labeling them to generate the original training data set and label set. II. Normalizing the original training data set and dividing it into three parts: training set, test set and validation set. III. Creation of networks and training. IV. Waiting for the training results and obtaining the training model. Since the problem faced is to determine the presence and location of insulator cracks, only a single label is required for labeling the data set. 
Table 1. RCNN structure and parameter design

\begin{tabular}{|c|c|c|c|c|c|c|c|c|c|}
\hline Input & Layer & Kernel & Kernel_num stri & ide pad & output & parame & ters & Memor & \\
\hline $512 \times 512 \times 3$ & CONV1 & $7 \times 17 \times 3$ & 24 & 0 & $169 \times 166 \times 24$ & 8.37 & K & 768.00 & $\mathrm{~K}$ \\
\hline $169 \times 166 \times 24$ & MAX POOL1 & $3 \times 3$ & 2 & 0 & $84 \times 83 \times 24$ & & & 658.81 & $\mathrm{~K}$ \\
\hline $84 \times 83 \times 24$ & NORM1 & & & & & & & 162.74 & $\mathrm{~K}$ \\
\hline $84 \times 83 \times 24$ & CONV2 & $5 \times 5 \times 24$ & 72 & 2 & $84 \times 83 \times 72$ & 42.19 & K & 162.74 & $\mathrm{~K}$ \\
\hline $84 \times 83 \times 72$ & MAX POOL2 & $3 \times 3$ & 2 & 0 & $42 \times 41 \times 72$ & & & 488.23 & K \\
\hline $42 \times 41 \times 72$ & NORM1 & & & & & & & 119.15 & $\mathrm{~K}$ \\
\hline $42 \times 41 \times 72$ & CONV3 & $3 \times 3 \times 72$ & 96 & 1 & $42 \times 41 \times 96$ & 60.75 & K & 119.15 & K \\
\hline $42 \times 41 \times 96$ & CONV4 & $3 \times 3 \times 96$ & 96 & 1 & $42 \times 41 \times 96$ & 81.00 & K & 158.86 & K \\
\hline $42 \times 41 \times 96$ & MAX POOL3 & $3 \times 3$ & 2 & 0 & $20 \times 20 \times 96$ & & & 158.86 & K \\
\hline $20 \times 20 \times 96$ & $\mathrm{FC} 1$ & & & & 1024 & 38717 & $\mathrm{~K}$ & 37.81 & $\mathrm{~K}$ \\
\hline 1024 & $\mathrm{FC} 2$ & & & & 1024 & 1024 & K & 1024.00 & $\mathrm{~K}$ \\
\hline 1024 & FC3-Classifier & & & & 1 & 1 & K & 1.00 & K \\
\hline 1024 & FC3-Regressor & & & & 512 & 262.14 & $\mathrm{~K}$ & 512.00 & $\mathrm{~K}$ \\
\hline
\end{tabular}

\section{Experimental Algorithm Analysis}

\subsection{Single crack picture recognition}

In the experiment, the insulator image acquisition with $512 \times 512$ resolution is performed by high precision industrial camera and other devices, and the information is transmitted to the backend system through the communication network. The server hardware device of the backend system is Intel corei5-6400 CPU and GTX1080 GPU. insulator crack detection algorithm is implemented in the backend system using Python. The prepared images to be predicted are pre-processed and normalized and then input to the trained $\mathrm{CNN}$, and the corresponding crack locations and FC layer output values are obtained and restored to the original input image to obtain the processed result maps. Finally, the result maps of each channel of R, G and B after processing are obtained as shown in figure 4.

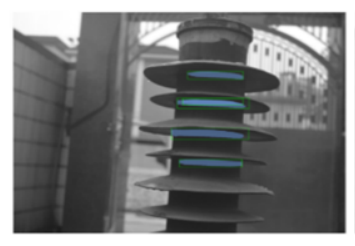

(a)-R-channel results

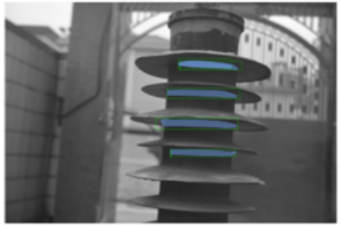

(b)- G-channel results 


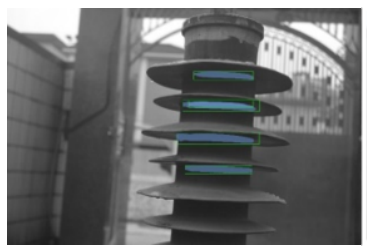

(c)-B-channel results

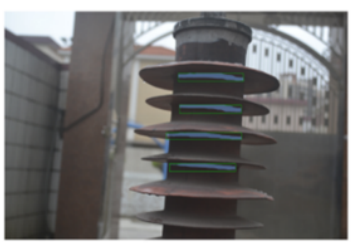

(d)-Output NMS result

Figure 4. Effect diagram of actual crack identification

Analysis of figures 4(a)(b)(c) shows that the components of each channel R, G, and $\mathrm{B}$ have accurately identified the crack defect site of the insulator, but the difference in the color of each channel as well as the channel component data leads to duplicate outputs in the detection structure of the $\mathrm{R}$ and $\mathrm{B}$ channels for the identification under the condition of the threshold value of 0.5 .

With the upper left corner of the image as the origin, the network output coordinate center, detection frame length and width, as well as the output defect confidence data of $\mathrm{R}, \mathrm{G}$ and $\mathrm{B}$ channels under the condition that the output pixel information is ignored and the threshold value is 0.5 . The output coordinate center $(x, y)$, frame length and width $(w, h)$, and the confidence data of each defect detection are shown in table 2 below after the frame de-weighting by NMS algorithm.

Table 2. Network detection output after deduplication

\begin{tabular}{llccl}
\hline Output list & Center(x,y) & Width Height( $\boldsymbol{w}, \boldsymbol{h})$ & Output confidence $>=50 . \%$ & $\begin{array}{l}\text { Output } \\
\text { source }\end{array}$ \\
\hline i. crack & $(1703,901)$ & $(759,107)$ & $67.99 \%$ & G-Channel \\
ii. crack & $(1822,615)$ & $(645,93)$ & $66.43 \%$ & R-Channel \\
iii. crack & $(1571,1206)$ & $(854,118)$ & $64.78 \%$ & R-Chnnel \\
iv. crack & $(1744,1535)$ & $(650,77)$ & $57.65 \%$ & G-Channel \\
\hline
\end{tabular}

Finally, the data obtained by using the Soft-NMS algorithm to remove the overlapping area of the output detection frame is drawn in the original image of the defect detection results, and the obtained image segmentation detection results are shown in blue labels in figure 4(d).

From figure 4, it can be seen that the cracked insulator defects of the three single channels of RGB were successfully detected using the R-CNN network, and the cracked areas were restored in the original cracked insulator pictures and marked in the original image. Meanwhile, since the final output of the network comes from the result of each channel after de-weighting by the NMS algorithm. Therefore, compared with the traditional single color channel image for prediction, using three single channels of RGB for insulator crack defect detection can improve the crack detection accuracy.

\subsection{Recognition Effect of 100 Cracked Pictures}

A test set containing 100 insulator images is constructed, with 95 images of insulators with cracks and 5 images of insulators without cracks. The trained network is tested and the results are as follows. 
Table 3. Original RCNN test results

\begin{tabular}{lccccc}
\hline $\begin{array}{l}\text { Photo } \\
\text { Gallery }\end{array}$ & Total images & Actual No. & RCNN Output & accuracy & Misjudging No. \\
\hline complete set & 100 & 242 & 227 & $93.80 \%$ & -17 \\
crack set & 95 & 242 & 226 & $93.39 \%$ & -16 \\
No-rack set & 5 & 0 & 1 & - & +1 \\
\hline
\end{tabular}

Table 4. Improved convolutional kernel RCNN test results

\begin{tabular}{llllll}
\hline Photo Gallery & Total images & Actual No. & RCNN Output & accuracy & Misjudging No. \\
\hline complete set & 100 & 242 & 241 & $99.59 \%$ & -1 \\
crack set & 95 & 242 & 240 & $99.17 \%$ & -2 \\
No-rack set & 5 & 0 & 0 & - & 0 \\
\hline
\end{tabular}

From table 3 and table 4, the recognition accuracy of RCNN with improved convolutional kernel is greater than $99 \%$, which is $5.8 \%$ higher than the original RCNN, proving that the improvement of convolutional kernel in this paper has significant improvement on the accuracy of crack detection.

\section{Conclusion}

Once the cracks or breakage of insulators are detected, they should be replaced immediately to avoid accidents. Based on the actual insulator crack image acquisition, this paper improves the convolution kernel and designs a set of R-CNN for composite insulator crack feature detection method. Experiments demonstrate that the detection method with the improved R-CNN network of convolutional kernel can achieve an accuracy of $99.6 \%$ for the detection of cracks with a small amount of image sample data. This contributes to the realization of non-contact, online monitoring of insulator crack defects and provides new ideas for fault diagnosis of transformer operating insulators, unmanned substations, and intelligent identification of cracked insulators by robots.

\section{References}

[1] Huang X, Chen G, Wang X, et al. Online monitoring and fault diagnosis of transmission lines [M]. 2nd Edition. Beijing: China Electric Power Press, 2014:206-232

[2] Qiu Z, Ruan J, Huang D, et al. Analysis and experimental study on aging form of transmission line suspension porcelain insulator [J]. High voltage technology, 2016, 42 (4): 1259-1267

[3] Ma min. insulator damage recognition based on image processing [D]. Shijiazhuang Railway University, 2019

[4] Wang Meng. Research on defect detection method based on insulator image [D]. Huazhong University of science and technology, 2019 